\title{
RESEARCH
}

Open Access

\section{GSTZ1 deficiency promotes hepatocellular carcinoma proliferation via activation of the KEAP1/NRF2 pathway}

Jingjing Li ${ }^{1,2+}$, Qiujie Wang ${ }^{1 \dagger}$, Yi Yang ${ }^{1}$, Chong Lei ${ }^{1}$, Fan Yang ${ }^{1}$, Li Liang ${ }^{1}$, Chang Chen ${ }^{3}$, Jie Xia ${ }^{1}$, Kai Wang ${ }^{1 *}$ and $\mathrm{Ni}$ Tang $^{1 *}$

\begin{abstract}
Background: Glutathione S-transferase zeta 1 (GSTZ1) is the penultimate enzyme in phenylalanine/tyrosine catabolism. GSTZ1 is dysregulated in cancers; however, its role in tumorigenesis and progression of hepatocellular carcinoma (HCC) is largely unknown. We aimed to assess the role of GSTZ1 in HCC and to reveal the underlying mechanisms, which may contribute to finding a potential therapeutic strategy against HCC.

Methods: We first analyzed GSTZ1 expression levels in paired human HCC and adjacent normal tissue specimens and the prognostic effect of GSTZ1 on HCC patients. Thereafter, we evaluated the role of GSTZ1 in aerobic glycolysis in HCC cells on the basis of the oxygen consumption rate (OCR) and extracellular acidification rate (ECAR). Furthermore, we assessed the effect of GSTZ1 on HCC proliferation, glutathione (GSH) concentration, levels of reactive oxygen species (ROS), and nuclear factor erythroid 2-related factor 2 (NRF2) signaling via gain- and loss- of GSTZ1 function in vitro. Moreover, we investigated the effect of GSTZ1 on diethylnitrosamine (DEN) and carbon tetrachloride $\left(\mathrm{CCl}_{4}\right)$ induced hepatocarcinogenesis in a mouse model of HCC.

Results: GSTZ1 was downregulated in HCC, thus indicating a poor prognosis. GSTZ1 deficiency significantly promoted hepatoma cell proliferation and aerobic glycolysis in HCC cells. Moreover, loss of GSTZ1 function depleted GSH, increased ROS levels, and enhanced lipid peroxidation, thus activating the NRF2-mediated antioxidant pathway. Furthermore, Gstz1 knockout in mice promoted DEN/CCl - -induced hepatocarcinogenesis via activation of the NRF2 signaling pathway. Furthermore, the antioxidant agent N-acetylcysteine and NRF2 inhibitor brusatol effectively suppressed the growth of Gstz1-knockout HepG2 cells and HCC progression in Gstz1 ${ }^{-1-}$ mice.
\end{abstract}

Conclusions: GSTZ1 serves as a tumor suppressor in HCC. GSH depletion caused by GSTZ1 deficiency elevates oxidative stress, thus constitutively activating the NRF2 antioxidant response pathway and accelerating HCC progression. Targeting the NRF2 signaling pathway may be a promising therapeutic approach for this subset of HCC.

Keywords: Glutathione S-transferase zeta 1, Hepatocellular carcinoma, Glutathione, Oxidative stress, KEAP1/NRF2 pathway

\footnotetext{
* Correspondence: wangkai@cqmu.edu.cn; nitang@cqmu.edu.cn

${ }^{\dagger}$ Jingjing Li and Qiujie Wang contributed equally to this work.

'Key Laboratory of Molecular Biology for Infectious Diseases (Ministry of

Education), Institute for Viral Hepatitis, Department of Infectious Diseases,

The Second Affiliated Hospital, Chongqing Medical University, Chongqing,

China

Full list of author information is available at the end of the article
}

(C) The Author(s). 2019 Open Access This article is distributed under the terms of the Creative Commons Attribution 4.0 International License (http://creativecommons.org/licenses/by/4.0/), which permits unrestricted use, distribution, and reproduction in any medium, provided you give appropriate credit to the original author(s) and the source, provide a link to the Creative Commons license, and indicate if changes were made. The Creative Commons Public Domain Dedication waiver (http://creativecommons.org/publicdomain/zero/1.0/) applies to the data made available in this article, unless otherwise stated. 


\section{Background}

Hepatocellular carcinoma (HCC) is one of the most common malignant tumors worldwide [1]. However, despite advancements in surgery and traditional chemotherapy, its overall prognosis is still poor, especially in advanced HCC. Similar to all malignant tumors, HCC is a multistep and multi-factorial disease. The Warburg effect, the first reported metabolic change in tumors, has provided novel insights into tumorigenesis, and metabolic reprogramming has been considered a new hallmark of cancer [2]. Hundreds of consistently deregulated metabolic genes have been identified in HCC in patients [3]. Restoration of these altered metabolic pathways may provide novel insights into cancer therapeutic approaches [4].

Glutathione $S$-transferase zeta 1 (GSTZ1), also known as maleylacetoacetate isomerase (EC 5.2.1.2), which catalyzes the glutathione-dependent isomerization of maleylacetoacetate (MAA) to fumarylacetoacetate (FAA) [5], is the penultimate enzyme in phenylalanine and tyrosine (Phe/Tyr) catabolism (Fig. 1a). Glutathione-S-transferase (GST) catalyzes the conjugation of glutathione to electrophilic endogenous or exogenous compounds, thus playing a critical role in cellular detoxification [6]. As a member of the GST family, GSTZ1 also catalyzes the oxygenation of dichloroacetic acid to glyoxylic acid and may be involved in xenobiotic $\alpha$-haloacid metabolism [7].

Gstz1-knockout mice have reportedly displayed rapid weight loss, renal and hepatic damage, necrosis, and lethality, when stressed with a high phenylalanine diet [8]. GSTZ1 was recently reported to be downregulated in HCC and upregulated in breast cancer [9], indicating that GSTZ1 dysregulation may be involved in tumorigenesis in humans. However, the underlying mechanism remains largely unknown.

As a consequence of metabolic and signaling aberrations, cancer cells generally have elevated levels of reactive oxygen species (ROS), which are balanced by an increased antioxidant capacity [10]. The nuclear factor erythroid 2related factor 2 (NRF2) is a master regulator of detoxification and the antioxidant response. NRF2 induces tumorigenesis, metastasis, and chemotherapeutic resistance [11]. Under normal conditions, NRF2 levels are retained at low levels by the E3 ubiquitin ligase Kelch-like ECHassociated protein 1 (KEAP1), which ubiquitinates NRF2 in the cytoplasm and targets it for degradation. Oxidative stress alters the conformation of KEAP1 via oxidation of cysteine residues, thus dissociating it from NRF2. NRF2 then translocates into the nucleus to activate its target genes, such as $\mathrm{NAD}(\mathrm{P}) \mathrm{H}$ : quinone oxidoreductase 1 (NQO1), heme oxygenase $(\mathrm{HO}-1)$, glutamate-cysteine ligase catalytic subunit (GCLC), and glutamate-cysteine ligase modifier subunit (GCLM), through binding with the antioxidant response element (ARE) in their promoter regions $[12,13]$.
In the present study, we aimed to investigate the role of GSTZ1 in HCC and elucidate the underlying mechanisms. We evaluated the role of GSTZ1 in aerobic glycolysis in HCC cells on the basis of the oxygen consumption rate (OCR) and extracellular acidification rate (ECAR) and assessed its effect on HCC proliferation, GSH concentration, ROS levels, and NRF2 signaling via gain and loss of GSTZ1 function in vitro. Furthermore, we investigated the effect of GSTZ1 on diethylnitrosamine (DEN) and $\mathrm{CCl}_{4}$-induced hepatocarcinogenesis in a mouse model of HCC. The present study may yield a potential therapeutic strategy against HCC.

\section{Methods}

Patient samples for gene and protein expression analysis Human HCC and adjacent non-tumor samples (surgical samples collected with informed consent from patients) were obtained from the first and second affiliated hospital of Chongqing Medical University. The samples were frozen immediately after surgery and stored in liquid nitrogen. The study was reviewed and approved by the Research Ethics Committee of Chongqing Medical University.

\section{Cell cultures}

Human hepatoma cell lines HepG2, SK-Hep1, and PLC/ $\mathrm{PRF} / 5$ were obtained from the American Type Culture Collection (Manassas, VA, USA). Other hepatoma cell lines including Huh7 and normal human hepatocytes MiHA were obtained from the Cell Bank of the Chinese Academy of Sciences (Shanghai, China). Apart from HepG2, cells were cultured in Dulbecco's modified Eagle's medium (Hyclone, Logan, UT, USA) supplemented with $10 \%$ fetal bovine serum (FBS; Gibco, Rockville, MD, USA), $100 \mathrm{IU}$ penicillin, and $100 \mathrm{mg} / \mathrm{ml}$ streptomycin. HepG2 cells were cultured in Minimum Essential medium (MEM, Hyclone). These cell lines have been recently authenticated through short tandem repeat profiling (Beijing Microread Gene Technology Co., Beijing, China). All cells were incubated in a humidified atmosphere at $37^{\circ} \mathrm{C}$ containing $5 \% \mathrm{CO}_{2}$.

\section{TCGA data analysis}

Gstz1 mRNA expression data were obtained from The Cancer Genome Atlas (TCGA) dataset and analyzed using Firebrowse (http://firebrowse.org/). To compare expression levels, we used RNA-Seq by Expectation Maximization to determine the transcript abundance of genes. Kaplan-Meier survival analysis was performed via patient stratification based on Gstz1 mRNA expression as high (top 33\%) or low (bottom 33\%). 


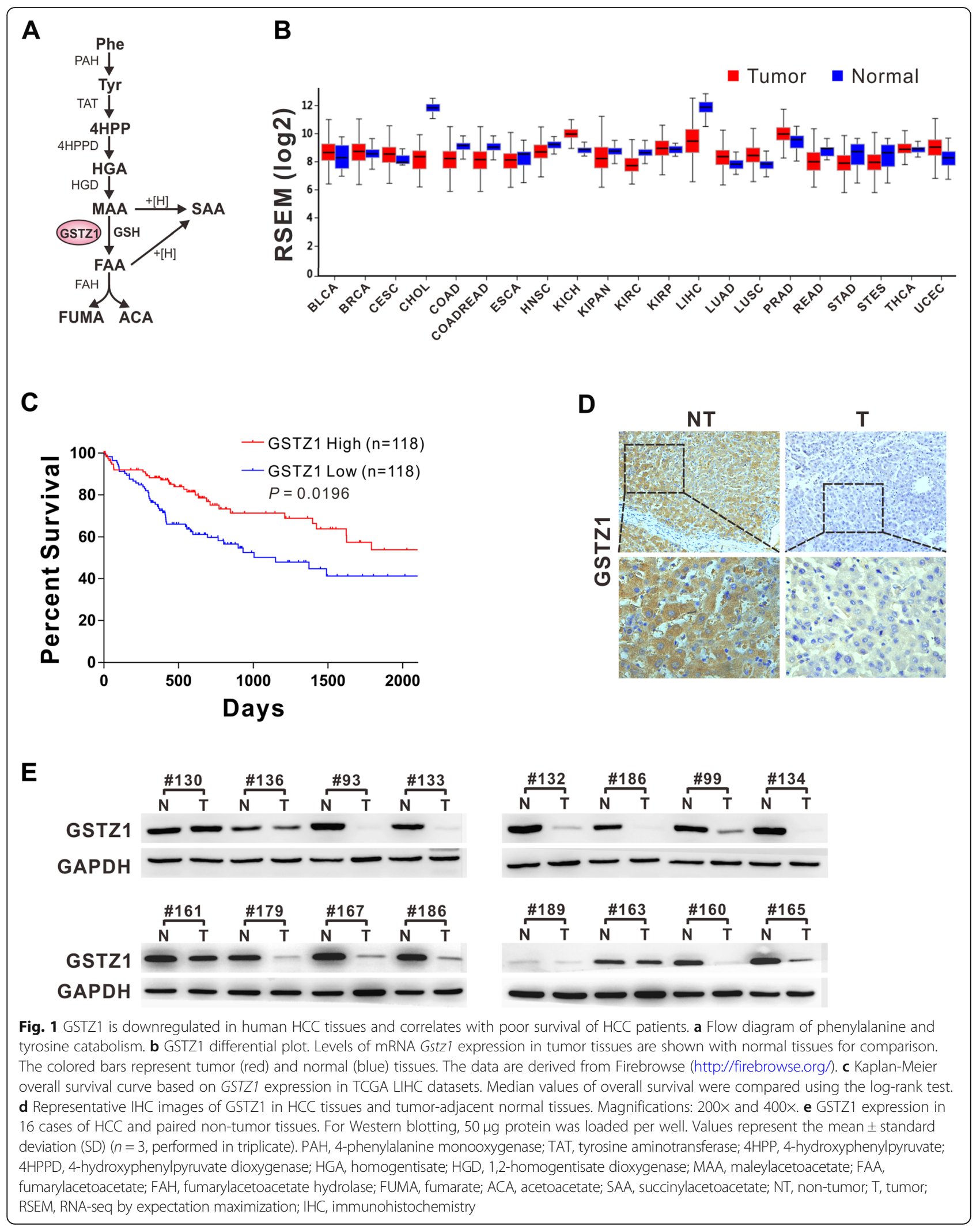


Construction of HCC cell lines overexpressing GSTZ1

The full-length cDNA of GSTZ1 (NM_145870.2) was amplified from plasmid pOTB7-GSTZ1 (FL09522; GeneCopoeia, Guangzhou, Guangdong, China) and inserted into the BamH I and Hind III sites of the shuttle vector pAdTrack-TO4 (from Dr. T-C He, University of Chicago, Chicago, IL, USA). Adenoviral recombinant pAd-GSTZ1 was generated using the AdEasy system [14]. HCC cell lines endogenously expressing GSTZ1 at low levels, including Huh7, SK-Hep1, and MHCC-97H, were infected with AdGSTZ1 to establish GSTZ1-overexpressing cell lines. An analogous adenovirus expressing only GFP (AdGFP) was used as the control.

\section{CRISPR-Cas9 mediated GSTZ1-knockout in HepG2 cells}

The E-CRISP online tool (http://www.e-crisp.org/ECRISP/designcrispr.html) was used to design the GSTZ1-targeting sequences. The 20-bp GSTZ1 targeting sequence selected herein, 5'- GCCCAGAACGCCAT CACTTG-3', immediately preceding a $5^{\prime}$-TGG-3' protospacer adjacent motif, was derived from exon 6 of GSTZ1. Thereafter, the synthesized oligos were annealed and cloned into lentiCRISPR v2 plasmid in accordance with the method of the Zhang lab (http://genome-engineering.org). The CRISPR/Cas9 plasmids lentiCRISPR v2, pMD2.G, and psPAX2 were kindly provided by Prof. Ding Xue from the School of Life Sciences, Tsinghua University (Beijing, China). LentiCRISPR v2, envelop plasmid pMD2.G, and packaging plasmid psPAX2 were co-transfected into HEK293T cells to generate lentiviruses, using lipofectamine 2000, in accordance with manufacturer's protocol. HepG2 cells were infected with the lentivirus, followed by puromycin selection to establish a stable GSTZ1-knockout (GSTZ1-KO) cell line. Thereafter, 96-well plates were used to select single-cell clones via the double dilution method. Clonal cell genomic DNA was extracted and cloned into the pMD19-T TA cloning vector and sequenced. The GSTZ1 knockout efficiency was confirmed through Western blotting.

\section{Quantitative reverse transcription polymerase chain reaction (qRT-PCR) analysis}

Total RNA was extracted using TRIzol (Invitrogen, Carlsbad, CA, USA) and reverse-transcribed to cDNA using PrimeScript RT reagent kit (Takara, Shiga, Japan) in accordance with the manufacturer's instructions. Among all primers used herein, only the qPCR primer for TXN was the exon-spanning type. To minimize genomic DNA contamination, all RNA samples were digested with RNase-free DNase (Promega, Madison, WI, USA) and re-purified using mini columns prior to reverse transcription and qPCR. Furthermore, we used a non-RT negative control to monitor the quality of the experiment. Real-time qPCR was performed to quantify
mRNA levels, using the iTaq Universal SYBR Green Supermix in accordance with the manufacturer's instructions. Each $10-\mu \mathrm{L}$ PCR reaction system comprised the following: $5 \mu \mathrm{L}$ SYBR Green Supermix, $0.5 \mu \mathrm{L}$ forward primer $(10 \mu \mathrm{mol} / \mathrm{L}), 0.5 \mu \mathrm{L}$ reverse primer $(10 \mu \mathrm{mol} / \mathrm{L})$, $2 \mu \mathrm{L} \mathrm{cDNA}$, and $2 \mu \mathrm{L}$ nuclease-free water. PCR was carried out using Bio-Rad CFX Connect Real-time PCR Detection System (Bio-Rad, Hercules, CA, USA) with the following reaction conditions: $95^{\circ} \mathrm{C}$ for $30 \mathrm{~s}$, followed by 35 cycles at $95^{\circ} \mathrm{C}$ for $10 \mathrm{~s}, 62^{\circ} \mathrm{C}$ for $30 \mathrm{~s}$, and $72{ }^{\circ} \mathrm{C}$ for $30 \mathrm{~s}$. Data were acquired during the extension step. The objective CT values were normalized to that of $\beta$-actin and the relative expression levels of genes were determined using the $\triangle \Delta C T$ method. The primer sequences and the accession numbers of individual genes are provided in Additional file 1: Table S1.

\section{Western blotting}

Total proteins were obtained from cells of tissues, using Cell lysis buffer for Western blotting and IP (Beyotime, Shanghai, China), containing 1\% of phenylmethanesulfonyl fluoride (PMSF) and cytoplasmic and nuclear proteins were extracted using the Nuclear and Cytoplasmic Protein Extraction Kit (Beyotime) in accordance with the manufacturer's instructions. The protein concentration of the homogenates was measured using the BCA protein assay Kit (Dingguo, Beijing, China) in accordance with the manufacturer's instructions. Protein lysates were boiled in SDS loading buffer for $5 \mathrm{~min}$. The same amount of proteins (30 to $50 \mu \mathrm{g}$ ) were separated via sodium dodecyl sulfatepolyacrylamide gel electrophoresis (8-10\% resolving gel) at $120 \mathrm{~V}$ for $60-90 \mathrm{~min}$. Thereafter, the proteins were electro-transferred onto a polyvinylidene difluoride membrane (Millipore, Boston, MA, USA). After blocking with $5 \%$ non-fat milk dissolved in TBST (10 mM Tris, $150 \mathrm{mM}$ $\mathrm{NaCl}$, and $0.1 \%$ Tween-20; $\mathrm{pH} 7.6$ ), the membranes were probed with the following primary antibodies overnight at $4{ }^{\circ} \mathrm{C}$ at a relative dilution rate in BSA: anti-GSTZ1 (1:1000; GTX106109, GeneTex, California, USA), anti-NQO1 (1: 1000; ab34173, Abcam, Cambridge, UK), anti-GAPDH (1: 5000; ab8245, Abcam), anti- $\beta$-actin (1:5000; ab6276, Abcam), anti-Lamin B1 (1:5000; ab133741, Abcam), anti$\beta$-tubulin (1:1000; ab108342, Abcam), anti-NRF2 (1:1000; ab62352, Abcam), and anti-4HNE (1:1000; ab46545, Abcam) antibodies. Thereafter, membranes were washed thrice in TBST, followed by incubation with the secondary antibody (1:5000 to 1:10000) conjugated with horseradish peroxidase (HRP) for $1 \mathrm{~h}$ at room temperature. Membranes were then washed thrice with TBST before enhanced chemiluminescence (ECL) exposure.

\section{Immunohistochemistry (IHC)}

The paraffin-embedded sections of tissues were incubated at $55^{\circ} \mathrm{C}$ for $2-4 \mathrm{~h}$, de-paraffinized in xylene for 20 
min, and rehydrated successively in $100,95,85$, and $75 \%$ ethanol and distilled water. After antigen retrieval with citrate buffer, the samples were incubated with 3\% hydrogen peroxide for $10 \mathrm{~min}$. Thereafter, samples were rinsed with PBS and blocked with goat serum for $1 \mathrm{~h}$ at room temperature. Residual serum was eliminated, and the samples were incubated with the following primary antibodies overnight at $4{ }^{\circ} \mathrm{C}$ at a relative dilution rate in PBS: anti-GSTZ1 (1:200), anti-NQO1 (1:200), and antiKi67 (1:100; ab15580, Abcam) antibodies. After rinsing with PBS, samples were incubated with reagent A of Elivision $^{\text {Tw }}$ plus Polyer HRP (Mouse/Rabbit) IHC Kit (Maixin, Fuzhou, China) for $20 \mathrm{~min}$, followed by incubation with reagent $\mathrm{B}$, secondary antibody conjugated with HRP for $30 \mathrm{~min}$. Signals indicating HRP activity were visualized using DAB reagent. The nuclei were counterstained with hematoxylin for $2.5 \mathrm{~min}$. After rinsing, the samples were dehydrated, treated with xylene for transparency, and observed using a microscope.

\section{Cell growth curve and clone formation assay}

The proliferation capacity of HCC cells was assessed using a cell growth curve and by assessing the clone formation capacity. Cells were seeded into a 96-well plate (2000-3000 cells/well), with three replicate wells per group. The cells were incubated in the ESCO Cell culture $\mathrm{CO}_{2}$ Incubator (Esco Technologies, Inc., Horsham, PA, USA) for $120 \mathrm{~h}$ and enumerated automatically every $24 \mathrm{~h}$. The growth curves were plotted using GraphPad Prism 7 software (GraphPad Software Inc., San Diego, CA, USA).

Cells were seeded in a 6-well plate (2000 cells/well), with three replicate wells per group, and cultured at $37^{\circ} \mathrm{C}$ and $5 \% \mathrm{CO}_{2}$. After 7-10 d, the cells were fixed with $4 \%$ paraformaldehyde for $30 \mathrm{~min}$ and incubated with Crystal Violet. After rinsing thrice with PBS, the clones were photographed and enumerated.

\section{EdU assay}

The proliferation capacity of HCC cells was assessed using the Cell-Light ${ }^{\mathrm{m}}$ EdU DNA cell proliferation kit (RiboBio, Guangzhou, Guangdong, China), in accordance with the manufacturer's instructions. Cells were seeded on coverslips in a 6-well plate. Before detection, cells were incubated with Reagent $\mathrm{A}$ at a dilution of 1 : 1000 in culture medium for $2 \mathrm{~h}$. After fixing with $4 \%$ paraformaldehyde, cells were incubated with fluorescent dye (Apollo 643) for $30 \mathrm{~min}$. DAPI was used to counterstain the nuclei. Thereafter, the coverslips were mounted on glass slides with Anti-fade Mounting Medium (Beyotime). Images were captured using a Leica confocal microscope (TSC SP8) with a 40× objective lens. The percentage of EdU-positive cells was determined for comparison.

\section{Quantification of oxygen consumption and the} extracellular acidification rate

The OCR and ECAR of Huh7 cells and HepG2 cells were determined using the Seahorse Bioscience Extracellular Flux Analyzer (XFe24; Seahorse Bioscience). Cells were plated at 50,000 cells/well with $250 \mu \mathrm{L}$ culture medium in a 24-well cell culture plate (Seahorse Bioscience), and incubated at $37^{\circ} \mathrm{C}, 5 \% \mathrm{CO}_{2}$ overnight. Thereafter, adherent cells were washed and replaced with $500 \mu \mathrm{L}$ XF Base Medium pH 7.4 (Seahorse Bioscience) supplemented with $1 \mathrm{mM}$ sodium pyruvate, 2 $\mathrm{mM}$ glutamine, and $10 \mathrm{mM}$ glucose. Cells were incubated at $37^{\circ} \mathrm{C}$ in a non- $\mathrm{CO}_{2}$ incubator for $1 \mathrm{~h}$. The hydrated XF24 sensor cartridge was loaded oligomycin ( $1 \mu \mathrm{M}$ final concentration) and protonophore trifluoromethoxy carbonyl cyanide phenylhydrazone (FCCP, $1 \mu \mathrm{M}$ final concentration). Thereafter, basal OCR and ECAR were measured in accordance with the manufacturer's instructions.

\section{ARE promotor activity}

The promotor activity of the antioxidative response element (ARE) was detected using the Dual-Luciferase Reporter System (Promega, Madison, WI, USA). The ARE reporter plasmid pGL3-ARE (see Additional file 2: Figure S1 for the plasmid map) was kindly provided by Prof. Yiguo Zhang from Chongqing University. Plasmids pGL3ARE $(3 \mu \mathrm{g})$ and pRL-TK $(0.3 \mu \mathrm{g})$ were co-transfected into cells cultured in a $6-\mathrm{cm}$ dish. After $24 \mathrm{~h}$, the cells were reseeded into 24-well plates, three replicate wells for each group. Thirty-six hours later, the cells were lysed with $1 \times$ Passive Lysis Buffer $(100 \mu \mathrm{L} /$ well $)$ for $15 \mathrm{~min}$ on shaker at room temperature. The lysates were centrifuged, and the supernatants were harvested. Thereafter, Firefly and Renilla luciferase activities were analyzed in accordance with the manufacturer's instructions. Relative luciferase activities, reflecting the promotor activities, were determined by normalizing firefly luciferase activity to that of the corresponding Renilla luciferase.

\section{$\mathrm{Gstz}^{-/-}$mouse in vivo assay}

Heterozygous 129-Gstz1 $1^{\mathrm{tm} 1 \mathrm{Jmf}} / \mathrm{Cnbc}$ mice (EM: 04481) were purchased from European Mouse Mutant Archive via Nanjing Biomedical Research Institute of Nanjing University and were crossed to breed wild-type (WT) and Gstz1 $1^{-/}$mice. Before hepatic GSH levels were determined via mass spectrometry, the mice were fed with $2 \%$ phenylalanine for 1 week. For subsequent analysis, mice were divided into groups as follows: WT (control), $\mathrm{Gstz1}^{-/-}, \mathrm{Gstz1}^{-/-}+\mathrm{NAC}$, and $\mathrm{Gstz1}^{-/-}+$Brusatol. Each group included ten mice, 5 female and 5 male. Mice of all groups were intraperitoneally administered DEN at $75 \mathrm{mg} / \mathrm{kg}$ at 2 weeks of age [15]. At the third week, the mice were fed with $0.25 \%$ Phe until euthanasia and 
intraperitoneally administered $\mathrm{CCl}_{4}$ at $2 \mathrm{~mL} / \mathrm{kg}$ twice a week for 12 weeks. At 20 weeks of age, another dose of DEN $(50 \mathrm{mg} / \mathrm{kg})$ was intraperitoneally administered. In the $G s t z 1^{-/-}+$NAC group, the Gstz1 $1^{-/-}$mice were administered $4 \mathrm{~g} / \mathrm{L} \mathrm{NAC}$ at 3 weeks until euthanasia. In the Gstz1 $1^{-/-}+$Brusatol group, the Gstz1 $1^{-/-}$mice were intraperitoneally administered brusatol at $2 \mathrm{mg} / \mathrm{kg}$ every $2 \mathrm{~d}$, 2 weeks before euthanasia. Their body weight was determined every week. Blood was sampled from the tail vein and serum $\alpha$-fetoprotein (AFP) levels were determined immediately before euthanasia. All mice were euthanized at 32 weeks of age, and their livers were dissected out. The liver weight and the number of hepatic tumors were determined. Protein and RNAs were extracted from hepatic tumors for Western blotting and qRT-PCR analysis, respectively. Hepatic tumors were fixed with $4 \%$ paraformaldehyde, embedded in paraffin, and sectioned for hematoxylin-eosin staining and immunohistochemistry. Hepatic tumor sections were further frozen for ROS detection. All animal procedures were performed in accordance with protocols approved by the Rules for Animal Experiments published by the Chinese Government and approved by the Research Ethics Committee of Chongqing Medical University (reference number: 2017010).

\section{Intracellular and in vivo ROS detection}

Cells were seeded on coverslips in a 6-well plate. After $24 \mathrm{~h}$, Cells were incubated with a fluorescence probe (CellROX Reagent Orange; Thermo, Wilmington, DE, USA) at a concentration of $5 \mu \mathrm{M}$ in $1 \times$ PBS for $30 \mathrm{~min}$ at $37^{\circ} \mathrm{C}$ (and protected against light). After washing thrice with $1 \times$ PBS, DAPI was used to counterstain the nuclei for $3 \mathrm{~min}$. Thereafter, cells were washed, and the coverslips were mounted on glass slides with Anti-fade Mounting Medium. Images were acquired using Leica Confocal Microscope 1 (TSC SP8) with a $60 \times$ oil immersion objective lens.

The frozen sections were prepared from hepatic tumors of 129 SvJ mice immediately after euthanasia. The frozen sections were gently washed with $1 \times$ PBS and incubated with a fluorescence probe (CellROX Reagent Orange; Thermo) at a concentration of $5 \mu \mathrm{M}$ in $1 \times \mathrm{PBS}$ for $30 \mathrm{~min}$ at $37^{\circ} \mathrm{C}$ (and protected against light). After three gentle washes with $1 \times$ PBS, DAPI was used to counterstain nuclei for $3 \mathrm{~min}$. Thereafter, the sections were washed and mounted with Anti-fade Mounting Medium. Images were acquired using Leica Confocal Microscope (TSC SP8) with a $20 \times$ objective lens.

\section{GSSG/GSH assay}

Cells were transfected with pEIGW Grx1-roGFP2 plasmid (Addgene \#64990). The intracellular ratio of oxidized GSH disulfide (GSSG) to reduced GSH (GSSG/
GSH) was detected as previously described [16]. Briefly, Grx1-roGFP2-expressing cells were excited with lasers at 405 and $488 \mathrm{~nm}$, followed by emission at 505-550 nm detected using a confocal microscope (Leica TSC SP8). Raw data were exported to ImageJ software (National Institutes of Health, Bethesda, MD, USA). GSSG/GSH ratios were determined through pixel-by-pixel division of the image acquired at $405 \mathrm{~nm}$ with that acquired at 488 $\mathrm{nm}$. Mouse liver tissues (approximately $100 \mathrm{mg}$ ) were used for GSH measurement. Liver tissues were extracted with cold acetonitrile/water (4:1, v/v) using a homogenizer, and GSH concentrations were measured via ultra-high performance liquid chromatography coupled with a triplequadrupole mass spectrometer (UHPLC-QqQ-MS).

\section{Statistical analysis}

Statistical analysis and data plotting were performed using GraphPad Prism 7 (GraphPad Software Inc., San Diego, CA, USA). Data were presented as mean \pm standard deviation (SD) values. Unless mentioned otherwise, the Student's $t$-test was used for between-group comparisons, and the one-way ANOVA analysis followed by the Tukey test for post hoc analysis was used for multiplegroup comparisons. Statistical significance was defined as $p<0.05$.

\section{Results}

\section{GSTZ1 downregulation in HCC predicts a poor patient} prognosis

To evaluate intratumor GSTZ1 deregulation, we compared its expression between tumor tissue and tumoradjacent normal tissue samples with mRNA-Seq data from TCGA database using Firebrowse (Fig. 1b). As shown in the differential plot, GSTZ1 was markedly downregulated in liver hepatocellular carcinoma (LIHC). We further evaluated the prognostic impact of GSTZ1 on patients with primary HCC in TCGA-LIHC. KaplanMeier's survival curve analysis revealed that patients with low GSTZ1 expression levels had a significantly shorter median overall survival than those with high expression (median survival, 1149 versus 2116 days, $p=0.0196$; Fig. 1c).

Further, we examined GSTZ1 expression in 16 paired clinical HCC and normal liver tissue samples via IHC and Western blotting, which revealed that GSTZ1 was significantly downregulated in HCC rather than tumoradjacent normal tissue (Fig. 1d-e). These data indicate that GSTZ1 downregulation in HCC may contribute to disease progression and predict a poor prognosis.

\section{GSTZ1 suppresses the proliferation of hepatoma cells}

To investigate the role of GSTZ1 in HCC, we first determined endogenous GSTZ1 protein levels in some hepatoma cell lines. Western blotting revealed that 


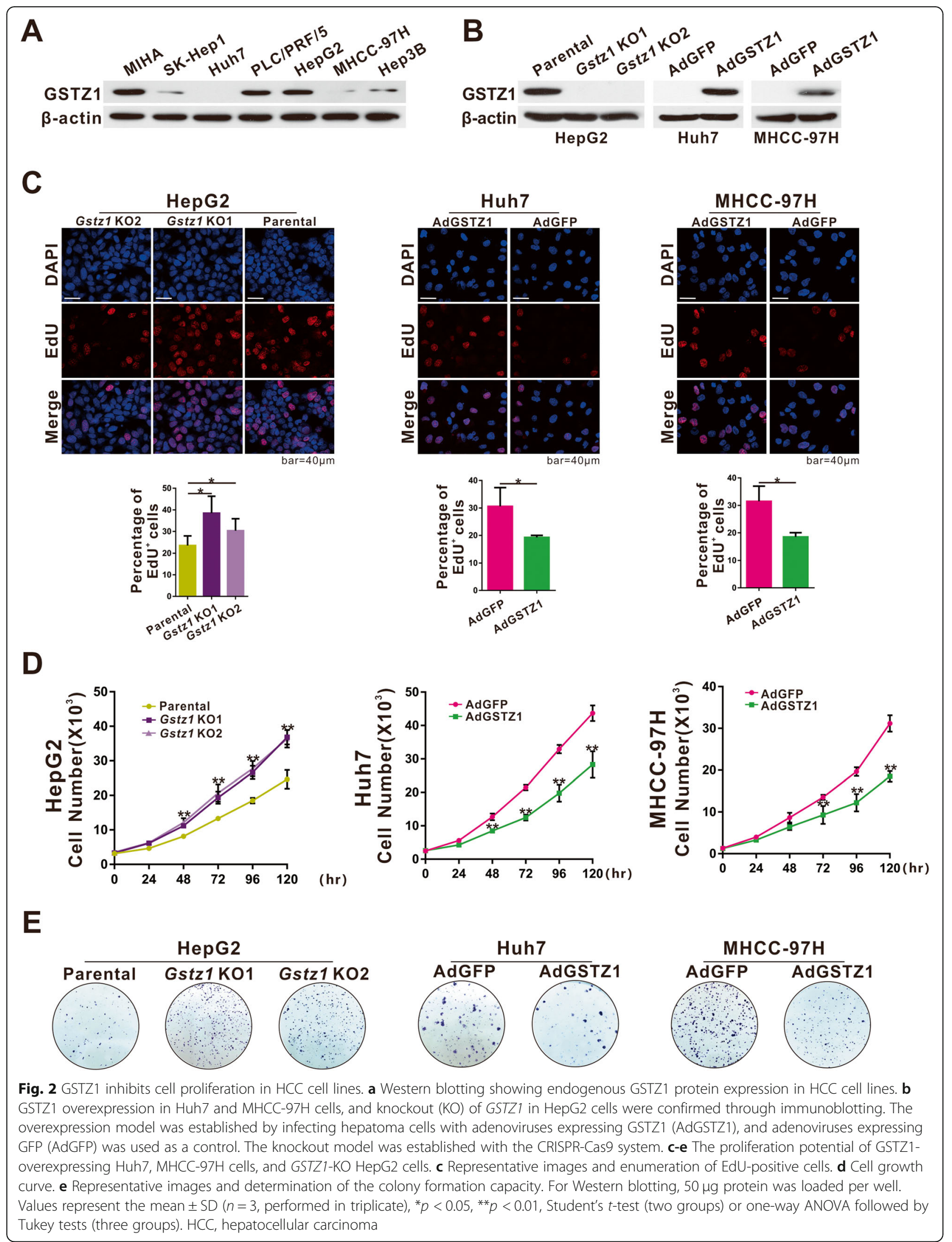




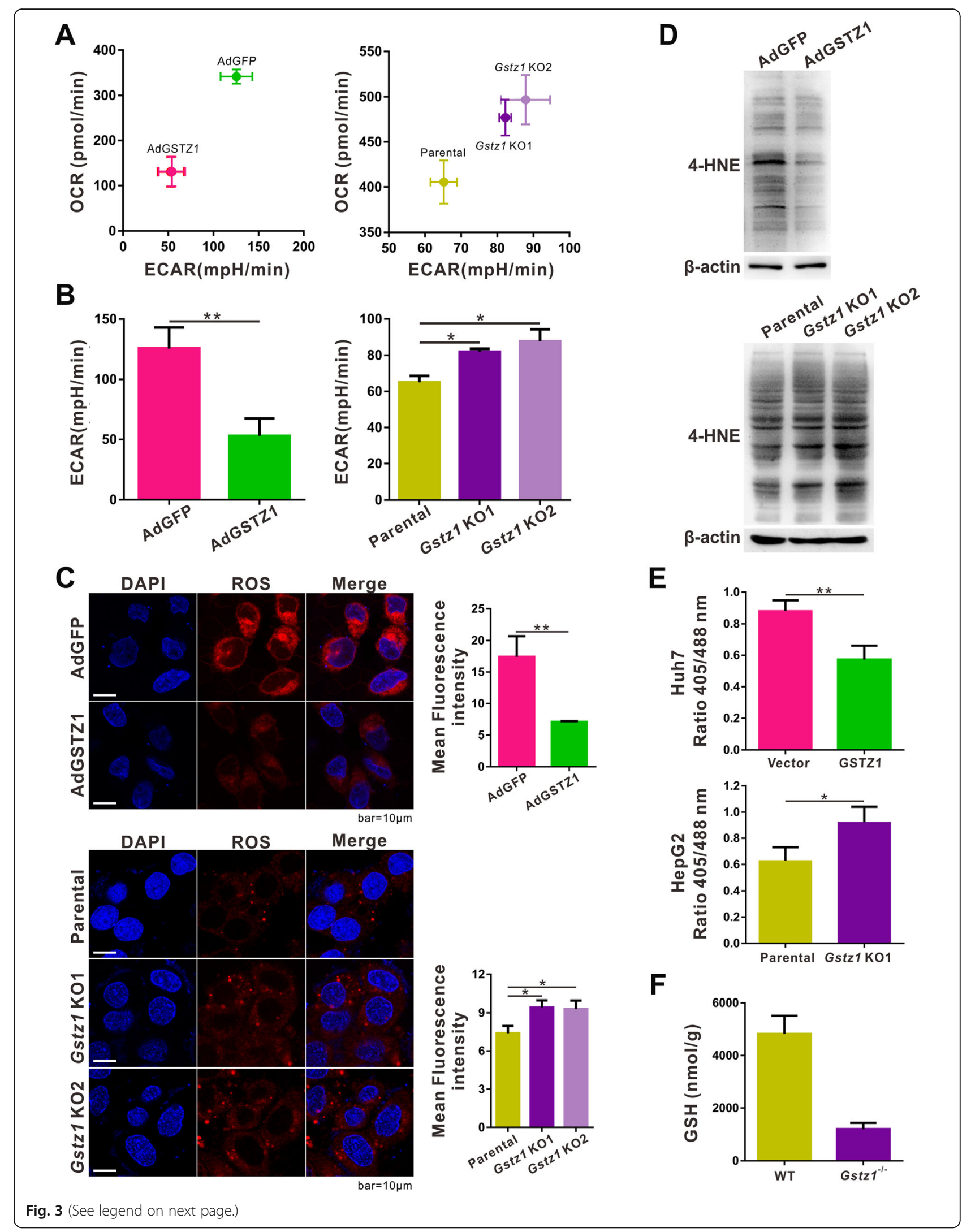


(See figure on previous page.)

Fig. 3 GSTZ1 suppresses the Warburg effect and ROS accumulation in HCC cell lines. a-b The ECAR and OCR in GSTZ1-overexpressing Huh7 cells (left) and GSTZ1-KO HepG2 cells (right) were determined using the Seahorse XF Cell Energy Phenotype test. GSTZ1-overexpressing Huh7 cells were less glycolytic than AdGFP control cells. In contrast, GSTZ1-KO HepG2 cells were more glycolytic than parental cells. a OCR versus ECAR. b ECAR values. c Representative fluorescence images (left) and quantification (right) of ROS levels in GSTZ1-overexpressing SK-Hep1 (top) and GSTZ1-KO HepG2 cells (bottom) stained with the CellROX Orange fluorescence probe. Immunofluorescence intensity was quantified using ImageJ. Values represent mean \pm SD values. $\mathbf{d}$ Western blotting revealed the levels of 4-HNE modification in proteins in GSTZ1-overexpressing Huh7 cells (top) and GSTZ1-KO HepG2 cells (bottom). e GSSG/GSH ratio for GSTZ1-overexpressing Huh7 cells (top) and GSTZ1-KO HepG2 cells (bottom) determined with Grx1-roGFP2. f Liver GSH levels in wildtype and Gstz1 ${ }^{-1-}$ mice, detected via mass spectrometry. For Western blotting, $50 \mu \mathrm{g}$ protein was loaded per well. Values represent mean \pm SD values $\left(n=3\right.$, performed in triplicate), ${ }^{*} p<0.05,{ }^{* *} p<0.01$, Student's $t$-test (two groups) or one-way ANOVA followed by Tukey tests (three groups). ECAR, extracellular acidification rate; OCR, oxygen consumption rate; HCC,

hepatocellular carcinoma; ROS; reactive oxygen species; GSH, glutathione; GSSG: GSH disulfide

GSTZ1 was substantially downregulated in most hepatoma cells, consistent with our previous study [14]. Among the HCC cell lines, Huh7, MHCC-97H, and SK-Hep1 cells expressed GSTZ1 at markedly lower levels than HepG2 and PLC/PRF/5 cells, wherein endogenous GSTZ1 expression was robust (Fig. 2a). To evaluate the effect of GSTZ1 on cell proliferation, we infected Huh7 and MHCC-97H cells with adenoviruses containing cDNA constructs encoding GSTZ1 (AdGSTZ1), and the empty adenovirus control (AdGFP). Furthermore, the stable HepG2 ${ }^{\text {Gstz1-/- }}$ cell line was established, in which the Gstz1 gene was knocked out using the CRISPR/cas9 system. Overexpression and knockout efficiencies were confirmed through Western blotting (Fig. 2b). GSTZ1 overexpression significantly suppressed the proliferation of both Huh7 and MHCC-97H cells, as revealed through EdU incorporation, cell growth curves, and colony formation assays, whereas GSTZ1 knockout promoted HepG2 cell proliferation (Fig. 2c-e).

\section{GSTZ1 suppresses the Warburg effect and ROS production in hepatoma cells}

Since GSTZ1 is a metabolic enzyme and most cancer cells perform aerobic glycolysis, we investigated whether GSTZ1 affects the metabolic state of hepatoma cells, using a Seahorse Bioscience XFe24 analyzer. Baseline OCR and ECAR were plotted as an energy map. Huh7 cells infected with AdGSTZ1 were less energetically active than those infected with the AdGFP control, whereas Gstz1-KO cells had a more active metabolic phenotype than parental HepG2 cells (Fig. 3a). Moreover, overexpression of GSTZ1 ( $p<0.01$, Fig. 3b left) significantly decreased the glycolytic rate of hepatoma cells, whereas Gstz1-KO ( $p<0.05$, Fig. 3b right) had the opposite effect.

ROS are byproducts of oxidative metabolism that are primarily produced in the mitochondria [17]. We examined ROS production in hepatoma cells with different GSTZ1 expression levels. ROS production was significantly reduced in GSTZ1-overexpressing SK-Hep1 cells $(p<0.01$, Fig. 3c, top) and significantly increased in
GSTZ1-KO HepG2 cells ( $p<0.05$, Fig. 3c bottom), compared to parental cells. A major electrophilic byproduct of lipid peroxidation, 4-Hydroxy-2-nonenal (4-HNE), is a biomarker of oxidative stress [18]. Concurrently, 4HNE-modified protein levels were reduced in GSTZ1overexpressing Huh7 cells and elevated in GSTZ1-KO HepG2 cells (Fig. 3d).

$\mathrm{GSH}$, the most abundant intracellular antioxidant, is oxidized to GSSG during oxidative stress [19]. To determine the effect of GSTZ1 on GSH levels in HCC cells, we determined the intracellular GSSG/GSH ratios with a redox-sensitive biosensor, Grx1-roGFP2. The GSSG/ GSH ratio was significantly decreased in GSTZ1-overexpressing Huh7 cells ( $p<0.01$, Fig. 3e top) and significantly increased in GSTZ1-KO HepG2 cells $(p<0.05$, Fig. 3e bottom). Similar results were observed with an animal model, and GSH levels in the liver of 129SvJ background $G s t z 1^{-/-}$mice were significantly lower than those in wildtype mice, as determined through mass spectrometry ( $p<0.01$, Fig. $3 \mathrm{f})$.

\section{Gstz1 deficiency activates the KEAP1/NRF2 pathway}

Gstz1-knockout mice reportedly exhibit increased oxidative stress and activation of NRF2-mediated antioxidant response pathways [20]. To verify this observation, we evaluated the effect of GSTZ1 on the NRF2 antioxidant pathway. The effect of GSTZ1 on the expression of AREdependent genes was analyzed using an ARE-regulated luciferase reporter plasmid (pGL3-ARE) in hepatoma cells. Moreover, the subcellular localization of NRF2 and mRNA expression of NRF2 target genes were evaluated. GSTZ1 overexpression decreased NRF2 nuclear translocation, ARE luciferase activity, and the expression of NRF2 target genes, including NQO1, HO-1, G6PD, GSTP1, GCLM, and TXN (Fig. 4a-c). In contrast, GSTZ1 knockout activated the NRF2 antioxidant pathway (Fig. 4a-c). To confirm this observation, we conducted RT-qPCR analysis using 40 tumor samples from HCC patients to detect GSTZ1 and NQO1 mRNA expression, and we observed a negative correlation between GSTZ1 and NQO1 expression ( $r=-0.37, p=0.0197$, Fig. $4 d$ ). Western blotting and IHC revealed increased levels of NQO1 and decreased 


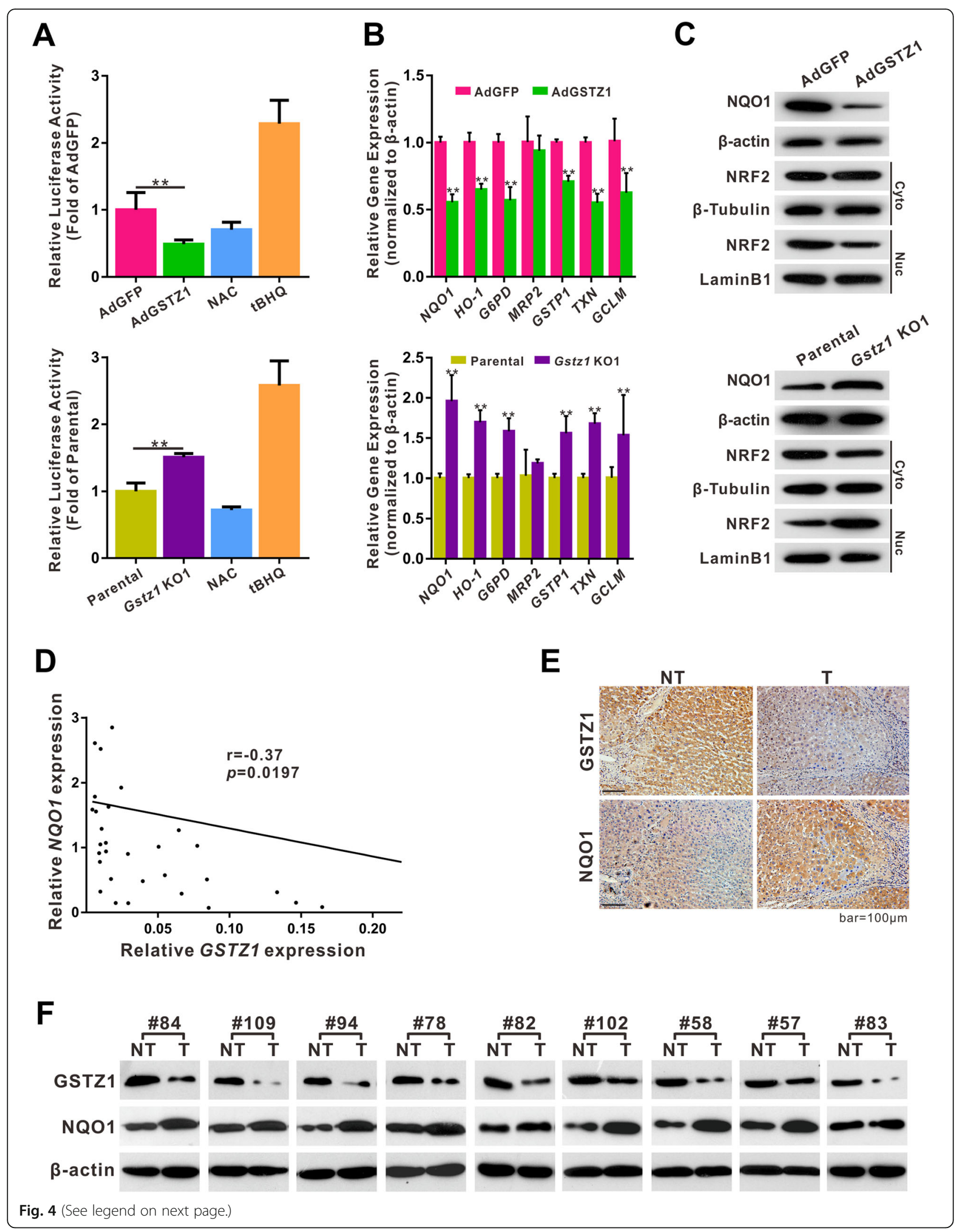


(See figure on previous page.)

Fig. 4 GSTZ1 negatively regulates the KEAP1/NRF2 pathway in human HCC and in liver tissue of mice. a-c ARE promoter activities in GSTZ1overexpressing Huh7 cells (left) and GSTZ1-KO HepG2 cells (right). a ARE promoter activities. The NRF2 activator tBHQ (100 $\mu M$ for $6 \mathrm{~h}$ ) and the antioxidant NAC ( $4 \mathrm{mM}$ for $24 \mathrm{~h}$ ) were used as positive and negative controls, respectively. b Relative mRNA expression of NRF2 target genes in GSTZ1-OE and GSTZ1-KO HCC cells, determined via GRT-PCR. c Western blotting to assess expression levels of NQO1, the NRF2 target, in total cell extracts, and of NRF2 in cytoplasmic and nuclear cell extracts, indicating the effect of GSTZ1 on NRF2 intracellular localization and transcription. $\beta$-actin, $\beta$-tubulin, and Lamin B1 served as reference proteins for total, cytoplasmic, and nuclear extracts, respectively. $\mathbf{d}$-f Association between GSTZ1 and NQO1 expression in HCC tissues. $\mathbf{d}$ Linear regression analysis showing the negative correlation between GSTZ1 and NQO1 mRNA expression in 40 cases of HCC ( $p=0.0197 ; r=-0.37$, Pearson correlation coefficient). e Representative immunohistochemistry images for GSTZ1 and corresponding NQO1 in HCC tissues and tumor-adjacent normal tissues. $\mathbf{f}$ Western blotting for GSTZ1 and NQO1 in 9 pairs of HCC and tumor-adjacent normal tissues. For Western blotting, $50 \mathrm{\mu g}$ protein was loaded per well. Values represent the mean $\pm \mathrm{SD}(n=3$, performed in triplicate), ${ }^{*} p<0.05,{ }^{* *} p<0.01$, Student's t-test. ARE, antioxidant response element; HCC, hepatocellular carcinoma; qRT-PCR, quantitative reverse transcription polymerase chain reaction

levels of GSTZ1 in most tumors, compared to tumoradjacent normal tissues (Fig. 4e and f). Together, these data indicate that GSTZ1 deficiency activates the KEAP1/ NRF2 pathway and promotes NQO1 expression in HCC.

Since oxidative stress and constitutive NRF2 activation are implicated in cancer cell growth and survival, modulation of the NRF2 antioxidant pathway has emerged as a promising approach for cancer therapy [1]. Therefore, we investigated whether the antioxidant agent $N$-acetylcysteine (NAC) or the NRF2 inhibitor brusatol (Bru) could suppress proliferation in GSTZ1-deficient hepatoma cells. As expected, NAC significantly reduced ROS levels ( $p<0.05$, Fig. 5a) in Gstz1KO HepG2 cells, whereas Bru significantly promoted ROS production $(p<0.01$, Fig. 5a). Both NAC and Bru suppressed the activation of the NRF2-mediated signaling pathway, albeit through different mechanisms. Colony formation, cell growth curves, and EdU incorporation assays revealed that both NAC and Bru significantly inhibited the proliferation of GSTZ1-KO HepG2 cells in vitro (Fig. 5b-d).

\section{Gstz1 knockout promotes $\mathrm{DEN} / \mathrm{CCl}_{4}$-induced hepatocarcinogenesis in mice}

To further investigate the role of GSTZ1 in hepatocarcinogenesis, wild-type (WT) and $G s t z 1^{-/-}$mice were injected with DEN $(75 \mathrm{mg} / \mathrm{kg})$, followed by repeated administration of $\mathrm{CCl}_{4}(2 \mathrm{~mL} / \mathrm{kg}$ twice a week for 12 weeks) and injected a second time with DEN $(50 \mathrm{mg} / \mathrm{kg})$ at 20 weeks of age (Figs. 6a and 7). All mice were administered $0.25 \%(\mathrm{w} / \mathrm{v})$ Phe in the drinking water from 3 weeks postpartum to increase the burden on the Phe/ Tyr catabolic pathway. Compared with WT mice, $\mathrm{Gstz1}^{-/-}$mice exhibited increased liver tumor sizes and numbers of tumor nodules (Fig. $6 \mathrm{~b}$ and c). Long-term administration of NAC water $(4 \mathrm{~g} / \mathrm{L}$ from 3 weeks postpartum until euthanasia) protected against tumorigenesis in $G_{s t z 1^{-1-}}$ mice (Fig. 6b and c). Bru-treated Gstz1 $1^{-1-}$ mice displayed smaller tumors and fewer tumor nodules than the untreated $G s t z 1^{-/-}$mice. Histological analysis indicated that the nuclei of liver tumors in $G s t z 1^{-1-}$ mice exhibited strong immunoreactivity for Ki67 (Fig. 6d). However, either NAC or Bru significantly inhibited cell proliferation in $\mathrm{Gstz1}^{-/-}$mice, which was highly consistent with the in vitro results.

Furthermore, Gstz1 $1^{-/}$mice exhibited enhanced ROS accumulation and 4-HNE modification in their liver tumor tissues, compared with WT mice (Fig. 6e and f). Consistent with the in vitro results, NAC reduced ROS levels, whereas Bru promoted ROS production in hepatic tumors in $\mathrm{Gstz1}^{-/-}$mice. NQO1 and nuclear NRF2 were markedly upregulated in hepatic tumors in $\mathrm{Gstz1}^{-1-}$ mice rather than in WT mice (Fig. $6 \mathrm{~g}$ and h), which indicated sustained activation of the KEAP1/NRF2 pathway caused by GSTZ1 deficiency.

Together, these data indicate that Gstz1 knockout increases the susceptibility to DEN/CCl 4 -induced hepatocarcinogenesis and progression in mice. GSTZ1 deficiency leads to GSH depletion, oxidative stress, and constitutive activation of the KEAP1/NRF2 pathway, thus accelerating the progression of hepatocarcinogenesis (Fig. 7).

\section{Discussion}

The liver is a key metabolic site of aromatic amino acids such as phenylalanine and tyrosine [21]; their catabolic pathway is of intrinsic value and clinical interest. Deficiencies in the enzymes of this catabolic pathway cause various severe diseases leading to hepatotoxicity and nephrotoxicity, such as tyrosinemia [22]. Hereditary tyrosinemia is caused by deficiencies in enzymes involved in phenylalanine and tyrosine catabolism, such as fumarylacetoacetate hydrolase, tyrosine aminotransferase, or 4-hydroxyphenylpyruvic dioxygenase. Hereditary tyrosinemia type 1 (HT1) is characterized by progressive liver diseases with an increased risk of HCC [23, 24]. However, limited information is available regarding the role of GSTZ1, the penultimate enzyme of phenylalanine and tyrosine catabolism, in diseases thus far. Recently, six children with mild hypersuccinylacetonemia caused by sequence variants in GSTZ1 were reported; however, no evidence of liver dysfunction was obtained [25].

The current study shows that GSTZ1 is markedly downregulated in HCC, thus predicting a poor prognosis, concurrent with recent reports $[3,9]$. In contrast, GSTZ1 is 
A
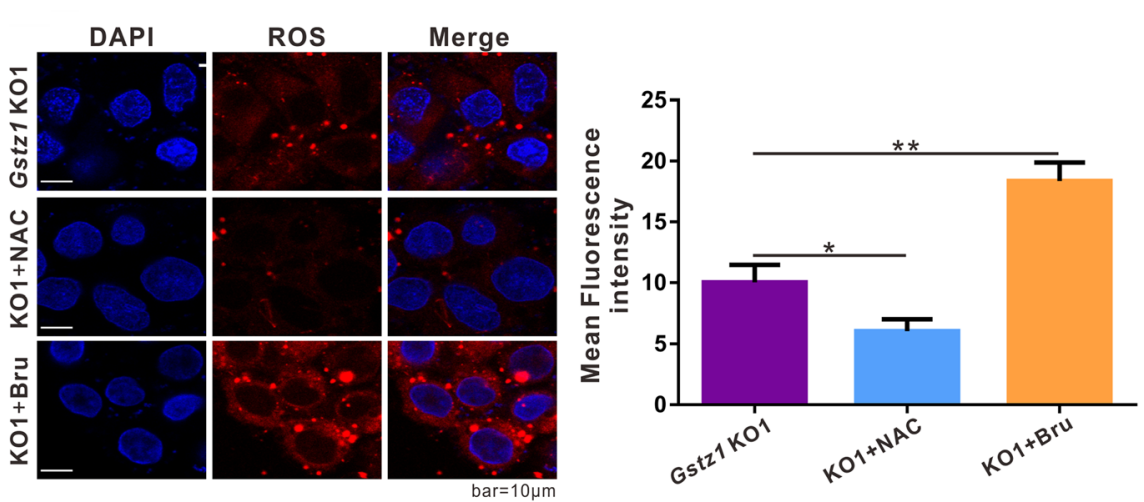

B

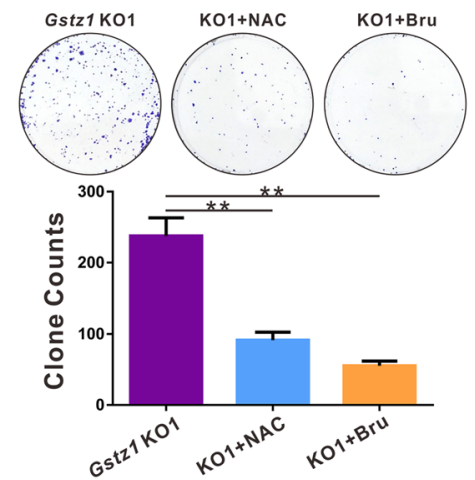

D
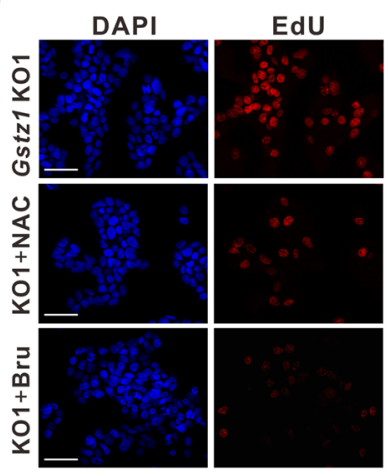

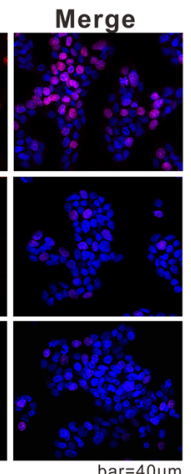

C

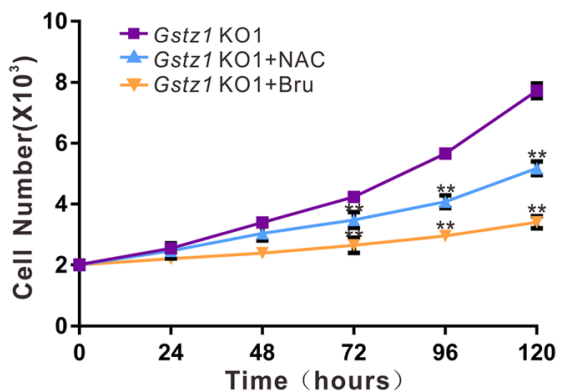

Fig. 5 GSTZ1 deficiency promotes HCC cell growth via activation of the KEAP1/NRF2 pathway. a ROS levels in Gstz1-KO1 cells treated with antioxidant NAC for $24 \mathrm{~h}$ or NRF2 inhibitor brusatol for $12 \mathrm{~h}$. b-d Effect of NAC or Bru on the proliferation potential of Gstz1-KO1 cells. b Representative images and colony formation potential. c Cell growth curve. $\mathbf{d}$ Representative images (left) and enumeration (right) of EdU positive cells. The final concentrations of NAC and brusatol were $4 \mathrm{mM}$ and $40 \mathrm{nM}$, respectively. Values represent means \pm SD $(n=3$, performed in triplicate), ${ }^{* *} p<0.01$, one-way ANOVA test. HCC, hepatocellular carcinoma; ROS, reactive oxygen species

reportedly upregulated in breast cancer [3, 9]. Several studies have highlighted dual roles for cancer-associated genes in different tumor types. Phosphoenolpyruvate carboxykinase, which converts oxaloacetate to phosphoenolpyruvate in the second step in gluconeogenesis, is reportedly upregulated in breast cancer, cervical carcinomas, and melanoma but downregulated in HCC [26]. Furthermore, GSTZ1 may play different roles in different tumor cell types, although limited information is available regarding its exact role in breast cancer [27, 28].
Our in vitro and in vivo gain- and loss-of-function studies revealed that GSTZ1 suppressed the Warburg effect and proliferation in hepatoma cells, indicating that GSTZ1 plays a tumor suppressor role in HCC. Our results suggest that GSTZ1 deficiency results in GSH depletion, followed by elevation of oxidative stress and sustained NRF2 activation, thus promoting proliferation in hepatoma cells. Intermediate metabolites of tyrosine catabolism accumulated in the urine of $\mathrm{Gstz1}^{-/-} 129 \mathrm{~Sv} 4$ mice [8] and in the serum of $\mathrm{Gstz1}^{-/-}$BALB/c mice [29]. 


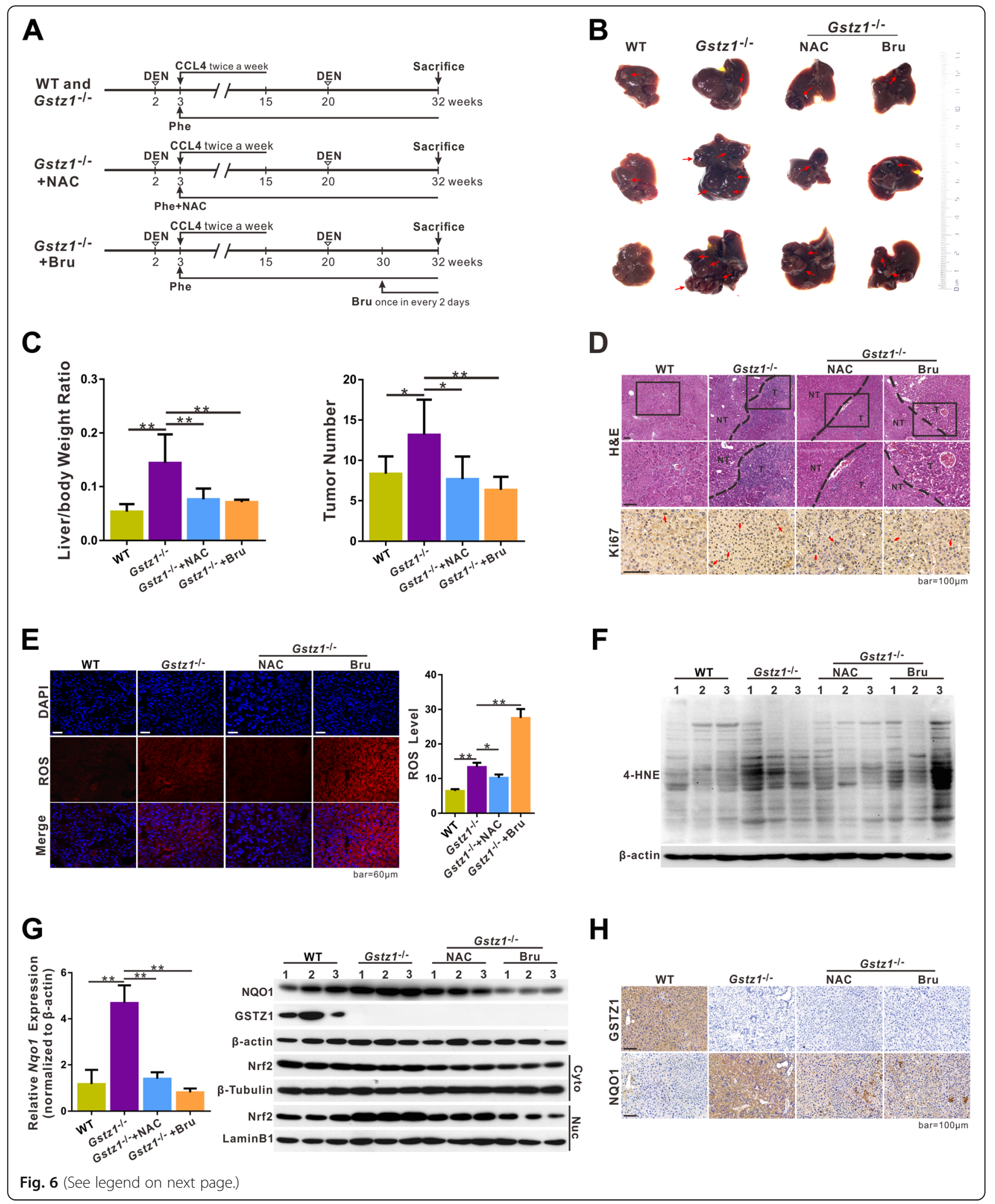


(See figure on previous page.)

Fig. 6 Knockout of Gstz1 promotes DEN/CCl 4 -induced hepatocarcinogenesis in vivo via activation of the KEAP1/NRF2 pathway. a Schematic representations of the experimental design for WT and Gstzl ${ }^{-/-}$mice. $\mathbf{b}$ Gross appearances of murine livers. Red arrows indicate tumors. $\mathbf{c}$ Liver/ body weight ratio (left) and number of tumors (right) in each group. $\mathbf{d}$ Representative H\&E staining and immunohistochemistry staining of Ki67 in liver tumors. e-f Oxidative stress levels in liver tumors. e Representative fluorescence staining of ROS with CellROX Orange probe in hepatic tumors of WT and Gstz7 ${ }^{--}$mice (left). Intracellular ROS quantification (right). f 4-HNE modification is all proteins in tumor tissues, analyzed via Western blotting. g NRF2 transcriptional activities in liver tumors. Relative Nqo1 mRNA expression, determined via qRT-PCR (Left). NQO1 expression in total cell extracts, and NRF2 expression in cytoplasmic and nuclear extracts, analyzed via Western blotting (Right). $\mathbf{h}$ Representative immunohistochemistry images of GSTZ1 and NQO1 in hepatic tumors. For Western blotting, $30 \mu \mathrm{g}$ protein was loaded per well. Values represent the mean \pm SD $\left(n=3\right.$, performed in triplicate), ${ }^{*} p<0.05,{ }^{* *} p<0.01$, Student's $t$-test (two groups) or one-way ANOVA followed by Tukey tests (four groups). H\&E, hematoxylin and eosin; DEN, diethylnitrosamine; HCC, hepatocellular carcinoma; ROS, reactive oxygen species; 4-HNE, 4-hydroxy-2nonenal; qRT-PCR, quantitative reverse transcription polymerase chain reaction

These metabolites react with GSH to form stable adducts and decrease intracellular GSH levels [30]. However, GSH can mediate the isomerization of MAA to FAA independent of GSTZ1 under a GSTZ1-deficient condition [8]. Herein, GSH depletion was demonstrated in the GSTZ1-KO hepatoma cells and the liver of $\mathrm{Gstz1}^{-/-}$mice, concurrent with our previous study on $\mathrm{Gstz1}^{-/-}$mice [20]. However, baseline GSH levels were observed in mice deficient for fumarate hydratase [31], leading to fumarate accumulation; however, this enzyme does not depend on GSH. Therefore, our results can be explained on the basis of GSH consumption caused by the co-effect of metabolites adduction and non-enzymatic bypass reactions in tyrosine catabolism.
ROS, such as the hydroxyl radical (HO•) and superoxide anion $\left(\mathrm{O}_{2}{ }^{-}\right)$, are generated by partial reduction of oxygen as byproducts of various cellular phenomena [32]. GSH is the most abundant antioxidant within cells and maintains cellular redox homeostasis. A reactive aldehyde, 4-HNE, is formed upon lipid peroxidation. Levels of global HNE-protein adducts are considered a biomarker for oxidative stress under various pathological conditions [33]. Herein, the generation of a constant level of oxidative stress, characterized by ROS and 4$\mathrm{HNE}$, in $\mathrm{Gstz1}^{-/-}$mice may have resulted from sustained GSH consumption.

Under physiological conditions, ROS are maintained at low levels and serve as signaling molecules to activate

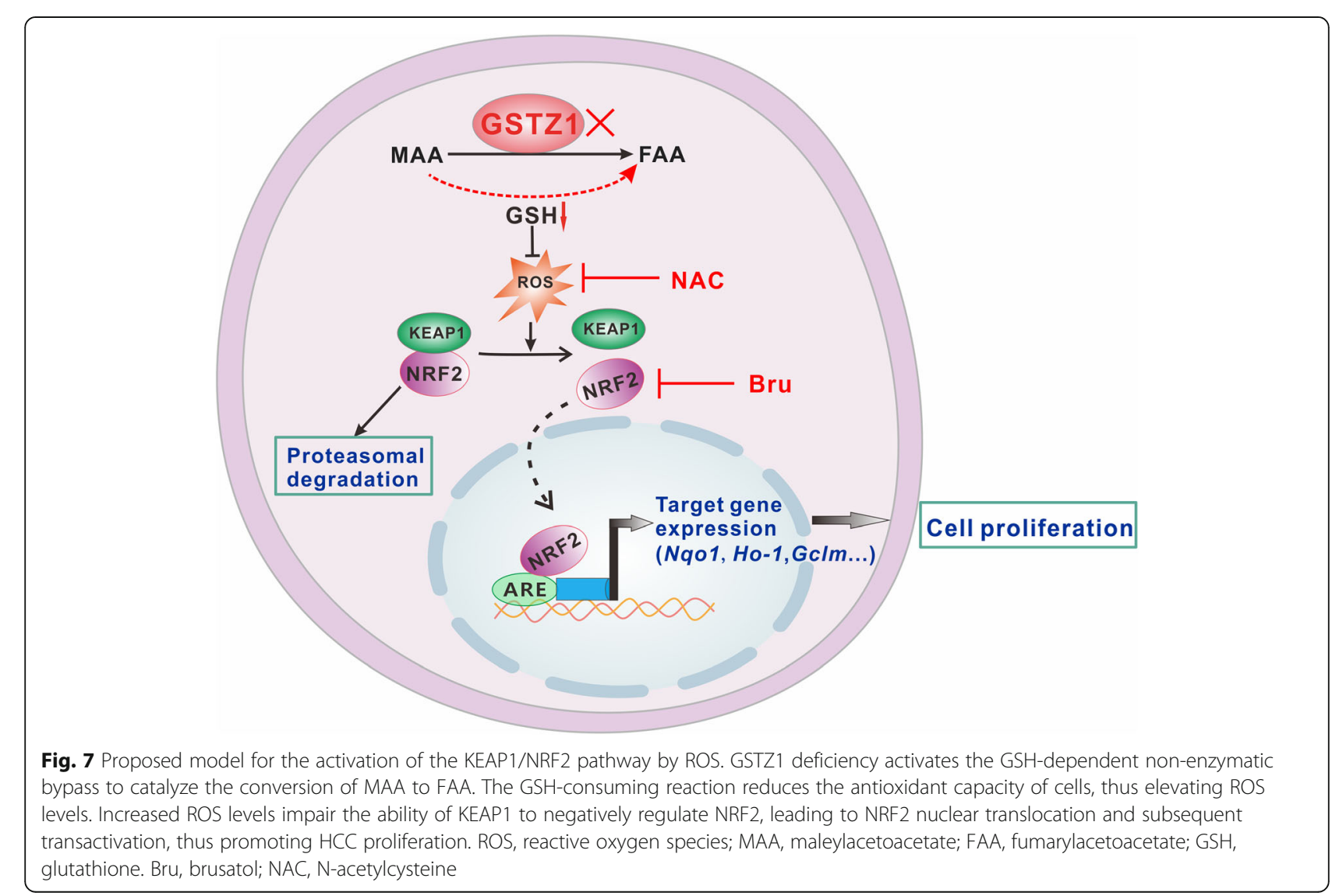


cell proliferation and survival pathways [34]. Moderate ROS levels induce oxidative stress, resulting in DNA damage and genetic instability and contribute to tumor initiation and progression [10]. However, once ROS levels become excessively high, they can lead to cell death [35]. Cancer cells exhibit increased intrinsic ROS levels owing to uncontrolled proliferation and a high metabolic rate [11]. Therefore, antioxidant pathways need to be further induced in tumor cells to maintain favorably high ROS levels for survival and proliferation [35]. This study shows that oxidative stress was significantly reduced in GSTZ1-overexpressing hepatoma cells but elevated in GSTZ1-KO hepatoma cells. Furthermore, Gstz1 $^{-/-}$mice exhibited enhanced ROS accumulation and 4-HNE modification in hepatic tumor tissues, compared with WT mice. These results indicate that GSTZ1 potentially plays a crucial role in regulating redox homeostasis in HCC.

NRF2 is a master regulator of the cellular antioxidant response. ROS activates NRF2 by promoting disulfide formation with KEAP1 [36]. Herein, GSTZ1 deficiency activated the NRF2 antioxidant pathway in vitro and in vivo. We speculate that the constant production of oxidative stress, resulting from GSTZ1 deficiency, may have activated the KEAP1/NRF2 pathway. NRF2 has been traditionally considered a tumor suppressor owing to its cytoprotective effects against damage from xenobiotics and oxidative stress. However, several studies have reported high constitutive activation of NRF2 in many tumors, such as carcinomas of the lungs, liver, gallbladder, ovary, breast, and stomach [37]. Concomitant to increased NRF2 activation, NRF2-target antioxidant enzymes such as GSTs and NQO1 are reportedly upregulated in cancer cells [38, 39]. NRF2 has recently been reported to promote cancer progression through metabolic reprogramming, apoptotic resistance, and metastasis promotion [11], further supporting our results that GSTZ1 deficiency promotes HCC proliferation and inhibition of the NRF2 pathway by NAC or Bru suppressed $\mathrm{Gstzl}^{-/-}$hepatoma cell proliferation in vitro and in vivo. The present results show increased ROS levels and enhanced activation of the NRF2 antioxidant pathway. This controversy also exists with respect to $\mathrm{FH}-$ deficient HLRCC cells [40, 41]; however, the underlying mechanism remains unknown. We speculate that the antioxidant capacity of NRF2 may not be adequate to scavenge all augmented ROS, thus increasing ROS levels, which was beneficial to tumor progression under these circumstances.

As a member of the GST family, GSTZ1 expression may be stimulated by NRF2 interacting with antioxidant response elements (AREs) [42], through which GSTZ1 would be upregulated. However, several other transcription factors along with NRF2 also interact with AREs to regulate target genes, such as $N R F 1, N R F 3$, and $B A C H 1$ [43]. Activation or suppression of target genes by NRF2 depends on dimerizing partners and AREs [43]. Moreover, NRF2 suppresses various targets [44]. No functional assays with AREs of GSTZ1 have been reported thus far; however, Marhenke et al. reported that NRF2 does not affect Gstz1 expression in the Fah/ $\mathrm{Nrf}^{-1-}$ mice model [45]. Furthermore, regulation of gene expression depends not only on transcriptional level, but also on epigenetic manipulation, such as DNA methylation and histone modifications [46]. Thus, whether NRF2 activation results in increased GSTZ1 expression or not needs further investigation. Our results indicate the importance of GSTZ1 on HCC proliferation through the KEAP1/NRF2 pathway. Accordingly, further studies are required to further elucidate the mechanisms underlying the inhibitory effect of GSTZ1 on HCC tumorigenesis and progression.

\section{Conclusions}

In summary, this study shows that GSTZ1 is downregulated in $\mathrm{HCC}$ and may serve as a prognostic marker. Our findings indicate that GSTZ1 deficiency induces oxidative stress, thus activating the KEAP1/NRF2 signaling pathway, which promotes HCC progression. This study shows the effect of GSTZ1 on HCC proliferation and the underlying mechanism, suggesting that modulation of oxidative stress and the NRF2 pathway may be a potential therapeutic strategy for this subset of HCC; however, further studies are required.

\section{Supplementary information}

Supplementary information accompanies this paper at https://doi.org/10. 1186/s13046-019-1459-6.

Additional file 1: Table S1. Quantitative RT-PCR Primer Sequences. Additional file 2: Figure S1. pGL3-ARE plasmid map.

\section{Abbreviations}

4-HNE: 4-hydroxy-2-nonenal; ARE: Antioxidant response element; DEN: Diethylnitrosamine; ECAR: Extracellular acidification rate; FAA: Fumarylacetoacetate; GSH: Glutathione; GSTZ1: Glutathione S-transferase zeta 1; HCC: Hepatocellular carcinoma; KEAP1: Kelch-like ECH-associated protein 1; LIHC: Liver hepatocellular carcinoma; MAA: Maleylacetoacetate; NAC: N-acetylcysteine; NRF2: Nuclear erythroid factor 2-related factor 2; OCR: Oxygen consumption rate; TCGA: The Cancer Genome Atlas

\section{Acknowledgments}

The authors wish to thank Dr. T.-C He (University of Chicago, USA) for providing the plasmids pAdEasy system and Prof. Ding Xue (School of Life Sciences, Tsinghua University) for supplying the CRISPR/Cas9 system, lentiCRISPR v2, pMD2.G and pSPAX2. We also thank Prof. Yiguo Zhang (Chongqing University) for providing the pGL3-ARE plasmid.

\section{Authors' contributions}

KW and NT conceived and designed the study. JJL and QJW performed most experiments and analyzed the data. $Y Y$ and $C L$ assisted with KEAP1/ NRF2 pathway analysis. FY and LL assisted with experiments in Gstz1knockout mice. CC assisted with GSH analysis. JX provided guidance and 
advice. JUL and NT drafted and edited the manuscript. All authors were involved in writing the paper and had final approval of the submitted versions.

\section{Funding}

This work was supported by the China National Natural Science Foundation (grant nos. 81872270 and 81572683 to N.T.; 81602417 to K.W.), the Major National S\&T program (2017ZX10202203-004 to N.T.), Natural Science Foundation Project of CQ CSTC (cstc2018jcyjAX0254 to N.T.), the Program for Innovation Team of Higher Education in Chongqing (CXTDX201601015), the Leading Talent Program of CQ CSTC (CSTCCXLJRC201719 to N.T.), the Natural Science Foundation Project of Chongqing (cstc2019jcyj-msxmX0587 to K.W.), and the Scientific Research Innovation Project for Postgraduate in Chongqing (grant no. CYS19192 and CYS18207).

\section{Availability of data and materials}

All data generated during this study are included in this article.

\section{Ethics approval and consent to participate}

All patients were provided informed consent, and this study was conducted with the approval of Institutional Ethical Review Board of Chongqing Medical University (project license number: 2017012). All animal experiments were conducted according to the guidelines of the Institutional Animal Care and Use Committee at Chongqing Medical University (The project license number: 2017012), and protocols of animal care and use adhere to National Regulations of the administration of laboratory animals.

\section{Consent for publication}

All contributing authors agree to the publication of this article.

\section{Competing interests}

The authors declare that they have no competing interest.

\section{Author details}

${ }^{1}$ Key Laboratory of Molecular Biology for Infectious Diseases (Ministry of Education), Institute for Viral Hepatitis, Department of Infectious Diseases, The Second Affiliated Hospital, Chongqing Medical University, Chongqing, China. ${ }^{2}$ Department of Blood Transfusion, The Second Affiliated Hospital, Chongqing Medical University, Chongqing, China. ${ }^{3}$ Institute of Life Sciences, Chongqing Medical University, Chongqing, China.

Received: 14 July 2019 Accepted: 17 October 2019

Published online: 30 October 2019

\section{References}

1. Menegon S, Columbano A, Giordano S. The dual roles of NRF2 in Cancer. Trends Mol Med. 2016;22(7):578-93.

2. Hainaut $P$, Plymoth A. Hallmarks of Cancer: the next generation. Curr Opin Oncol. 2013;25(1):50-1.

3. Nwosu ZC, Megger DA, Hammad S, et al. Identification of the consistently altered metabolic targets in human hepatocellular carcinoma. Cell Mol Gastroenterol Hepatol. 2017:4(2):303-323.e1.

4. Vander HMG. Targeting cancer metabolism: a therapeutic window opens. Nat Rev Drug Discov. 2011:10(9):671-84

5. Fernández-Cañón JM, Peñalva MA. Characterization of a fungal maleylacetoacetate isomerase gene and identification of its human homologue. J Biol Chem. 1998;273(1):329-37.

6. Allocati N, Masulli M, Di IC, Federici L. Glutathione transferases: substrates, inihibitors and pro-drugs in cancer and neurodegenerative diseases. Oncogenesis. 2018;7(1):8

7. Tong Z, Board PG, Anders MW. Glutathione transferase zeta catalyses the oxygenation of the carcinogen dichloroacetic acid to glyoxylic acid. Biochem J. 1998:331(Pt 2):371-4.

8. Fernández-Cañón JM, Baetscher MW, Finegold M, Burlingame T, Gibson KM, Grompe M. Maleylacetoacetate isomerase (MAAI/GSTZ)-deficient mice reveal a glutathione-dependent nonenzymatic bypass in tyrosine catabolism. Mol Cell Biol. 2002;22:4943-51.

9. Jahn SC, Solayman MH, Lorenzo RJ, Langaee T, Stacpoole PW, James MO. GSTZ1 expression and chloride concentrations modulate sensitivity of cancer cells to dichloroacetate. Biochim Biophys Acta. 2016;1860(6):1202-10.
10. Gorrini C, Harris IS, Mak TW. Modulation of oxidative stress as an anticancer strategy. Nat Rev Drug Discov. 2013;12(12):931-47.

11. de la Vega MR, Chapman E, Zhang DD. NRF2 and the hallmarks of Cancer. Cancer Cell. 2018:34(1):21-43.

12. Hayes AJ, Skouras C, Haugk B, Charnley RM. Keap1-Nrf2 signalling in pancreatic cancer. Int J Biochem Cell Biol. 2015;65:288-99.

13. Nioi $P$, McMahon M, Itoh K, Yamamoto M, Hayes JD. Identification of a novel Nrf2-regulated antioxidant response element (ARE) in the mouse $\mathrm{NAD}(\mathrm{P}) \mathrm{H}$ :quinone oxidoreductase 1 gene: reassessment of the ARE consensus sequence. Biochem J. 2003;374(Pt 2):337-48.

14. Yang F, Li J, Deng H, Wang Y, Lei C, Wang Q, et al. GSTZ1-1 Deficiency Activates NRF2/IGF1R Axis in HCC via Accumulation of Oncometabolite Succinylacetone. EMBO J. 2019;38(15):e101964.

15. Shiota G, Harada K, Ishida M, Tomie Y, Okubo M, Katayama S, Ito H, Kawasaki H. Inhibition of hepatocellular carcinoma by glycyrrhizin in diethylnitrosamine-treated mice. Carcinogenesis. 1999;20:59-63.

16. Gutscher M, Pauleau AL, Marty L, et al. Real-time imaging of the intracellular glutathione redox potential. Nat Methods. 2008;5(6):553-9.

17. Sabharwal SS, Schumacker PT. Mitochondrial ROS in cancer: initiators, amplifiers or an Achilles' heel. Nat Rev Cancer. 2014;14(11):709-21.

18. Zhong $\mathrm{H}_{\text {, }}$ Yin $\mathrm{H}$. Role of lipid peroxidation derived 4-hydroxynonenal (4HNE) in cancer: focusing on mitochondria. Redox Biol. 2015;4:193-9.

19. Meister A. Selective modification of glutathione metabolism. Science. 1983; 220(4596):472-7.

20. Blackburn AC, Matthaei KI, Lim C, Taylor MC, Cappello JY, Hayes JD, Anders MW, Board PG. Deficiency of glutathione transferase zeta causes oxidative stress and activation of antioxidant response pathways. Mol Pharmacol. 2006:69:650-7.

21. Tessari P, Vettore M, Millioni R, Puricelli L, Orlando R. Effect of liver cirrhosis on phenylalanine and tyrosine metabolism. Curr Opin Clin Nutr Metab Care. 2010;13(1):81-6.

22. Chakrapani A, Gissen P, McKiernan P. Disorders of tyrosine metabolism. In: Inborn Metabolic Diseases. Berlin, Heidelberg: Springer; 2012. p. 265-6.

23. van Spronsen FJ, Thomasse Y, Smit GP, et al. Hereditary tyrosinemia type I: a new clinical classification with difference in prognosis on dietary treatment. Hepatology. 1994;20(5):1187-91.

24. Weinberg AG, Mize CE, Worthen HG. The occurrence of hepatoma in the chronic form of hereditary tyrosinemia. J Pediatr. 1976;88(3):434-8.

25. Yang H, Al-Hertani W, Cyr D, et al. Hypersuccinylacetonaemia and normal liver function in maleylacetoacetate isomerase deficiency. J Med Genet. 2017;54(4):241-7.

26. Wang Z, Dong C. Gluconeogenesis in Cancer: function and regulation of PEPCK, FBPase, and G6Pase. Trends Cancer. 2019;5:30-45.

27. Smith RA, Curran JE, Weinstein SR, Griffiths LR. Investigation of glutathione S-transferase zeta and the development of sporadic breast cancer. Breast Cancer Res. 2001;3:409-11.

28. Andonova IE, Justenhoven C, Winter S, Hamann U, Baisch C, Rabstein S, Spickenheuer A, Harth V, Pesch B, Brüning T, et al. No evidence for glutathione S-transferases GSTA2, GSTM2, GSTO1, GSTO2, and GSTZ1 in breast cancer risk. Breast Cancer Res Treat. 2010;121:497-502.

29. Lim CE, Matthaei Kl, Blackburn AC, et al. Mice deficient in glutathione transferase zeta/maleylacetoacetate isomerase exhibit a range of pathological changes and elevated expression of alpha, mu, and pi class glutathione transferases. Am J Pathol. 2004;165(2):679-93.

30. Langlois $C$, Jorquera $R$, Orejuela $D$, et al. Rescue from neonatal death in the murine model of hereditary tyrosinemia by glutathione monoethylester and vitamin C treatment. Mol Genet Metab. 2008;93(3):306-13.

31. Adam J, Hatipoglu E, O'Flaherty L, et al. Renal cyst formation in Fh1deficient mice is independent of the Hif/Phd pathway: roles for fumarate in KEAP1 succination and Nrf2 signaling. Cancer Cell. 2011;20(4):524-37.

32. Pelicano H, Carney D, Huang P. ROS stress in cancer cells and therapeutic implications. Drug Resist Updat. 2004;7(2):97-110.

33. Guéraud F. 4-Hydroxynonenal metabolites and adducts in pre-carcinogenic conditions and cancer. Free Radic Biol Med. 2017:111:196-208.

34. Panieri E, Santoro MM. ROS homeostasis and metabolism: a dangerous liason in cancer cells. Cell Death Dis. 2016;7(6):e2253.

35. Cairns RA, Harris IS, Mak TW. Regulation of cancer cell metabolism. Nat Rev Cancer. 2011;11:85-95.

36. Fourquet S, Guerois R, Biard D, Toledano MB. Activation of NRF2 by nitrosative agents and $\mathrm{H} 2 \mathrm{O} 2$ involves KEAP1 disulfide formation. J Biol Chem. 2010;285(11):8463-71. 
37. Sporn MB, Liby KT. NRF2 and cancer: the good, the bad and the importance of context. Nat Rev Cancer. 2012;12(8):564-71.

38. Di PG, Magno LA, Rios-Santos F. Glutathione S-transferases: an overview in cancer research. Expert Opin Drug Metab Toxicol. 2010;6:153-70.

39. Belinsky M, Jaiswal AK. NAD(P)H:quinone oxidoreductase1 (DT-diaphorase) expression in normal and tumor tissues. Cancer Metastasis Rev. 1993;12: 103-17.

40. Sudarshan S, Sourbier C, Kong HS, Block K, Valera RVA, Yang Y, Galindo C, Mollapour M, Scroggins B, Goode N, et al. Fumarate hydratase deficiency in renal cancer induces glycolytic addiction and hypoxia-inducible transcription factor 1alpha stabilization by glucose-dependent generation of reactive oxygen species. Mol Cell Biol. 2009;29:4080-90.

41. Ooi A, Wong JC, Petillo D, Roossien D, Perrier-Trudova V, Whitten D, Min BW, Tan MH, Zhang Z, Yang XJ, et al. An antioxidant response phenotype shared between hereditary and sporadic type 2 papillary renal cell carcinoma. Cancer Cell. 2011;20:511-23.

42. Itoh K, Chiba T, Takahashi S, Ishii T, Igarashi K, Katoh Y, Oyake T, Hayashi N, Satoh K, Hatayama I, et al. An Nrf2/small Maf heterodimer mediates the induction of phase II detoxifying enzyme genes through antioxidant response elements. Biochem Biophys Res Commun. 1997;236:313-22.

43. Raghunath A, Sundarraj K, Nagarajan R, Arfuso F, Bian J, Kumar AP, Sethi G, Perumal E. Antioxidant response elements: discovery, classes, regulation and potential applications. Redox Biol. 2018;17:297-314.

44. Tonelli C, IIC C, Tuveson DA. Transcriptional regulation by Nrf2. Antioxid Redox Signal. 2018;29:1727-45.

45. Marhenke $S$, Lamlé J, Buitrago-Molina LE, Cañón JM, Geffers R, Finegold M, Sporn M, Yamamoto M, Manns MP, Grompe M, et al. Activation of nuclear factor E2-related factor 2 in hereditary tyrosinemia type 1 and its role in survival and tumor development. Hepatology. 2008;48:487-96.

46. Jaenisch R, Bird A. Epigenetic regulation of gene expression: how the genome integrates intrinsic and environmental signals. Nat Genet. 2003; 33(Suppl):245-54.

\section{Publisher's Note}

Springer Nature remains neutral with regard to jurisdictional claims in published maps and institutional affiliations.

Ready to submit your research? Choose BMC and benefit from:

- fast, convenient online submission

- thorough peer review by experienced researchers in your field

- rapid publication on acceptance

- support for research data, including large and complex data types

- gold Open Access which fosters wider collaboration and increased citations

- maximum visibility for your research: over $100 \mathrm{M}$ website views per year

At BMC, research is always in progress.

Learn more biomedcentral.com/submissions 